\title{
Description of the critical point symmetry in 124Te by IBM-2
}

\author{
DaLi Zhang ${ }^{1, *}$ and ChengFu $\mathrm{Mu}^{1}$ \\ ${ }^{1}$ Department of physics, Huzhou University, Huzhou 313000, Zhejiang, China
}

(Dated: December 10, 2018)

\begin{abstract}
Based on the neutron and proton degrees of freedom, the low-lying energy levels, the $E 2, M 1$ and E0 transition strengths of nucleus ${ }^{124} \mathrm{Te}$ have been calculated by the neutron-proton interacting boson model. The calculated results are quite consistent with the experimental data. By comparing the key observables of states at the critical point of $\mathrm{U}_{\pi \nu}(5)-\mathrm{O}_{\pi \nu}(6)$ transition with experimental data and calculated results, we show that the ${ }^{124} \mathrm{Te}$ is a possible nucleus at the critical point of the second order phase transition from vibration to unstable rotation and such a critical point contains somewhat triaxial rotation. The $0_{2}^{+}$state of ${ }^{124} \mathrm{Te}$ can be interpreted as the lowest state of the first excited family of intrinsic levels in the critical point symmetry.
\end{abstract}

Key words: ${ }^{124} \mathrm{Te}$, low-lying structure, the critical point, $\mathrm{U}_{\pi \nu}(5)-\mathrm{O}_{\pi \nu}(6)$ transition.

PACS numbers: 21.10.Re, 21.60.Fw, 27.60.+j

*Corresponding author zdl@zjhu.edu.cn 


\section{INTRODUCTION}

How to characterize the underlying structure of ${ }^{124} \mathrm{Te}$ is a difficult task and has attracted considerable interests in variety of theoretical and experimental studies [1 6$]$. The ${ }^{124}$ Te lies outside the major shell $Z=50$ with two protons, and its ratio $E\left(4_{1}^{+} / 2_{1}^{+}\right)=2.07$ is very close to the spherical vibrator value of 2.00 . This indicates that the ${ }^{124} \mathrm{Te}$ is a vibrational or U(5) symmetric nucleus. However, the energy levels spread of the two-phonon multiplets do not suggest a vibrational character in ${ }^{124} \mathrm{Te}$. Meanwhile, the energy level of the lowest excited $0^{+}$state suggests that the ${ }^{124}$ Te exhibits more $\gamma$-unstable or $\mathrm{O}(6)$ behavior, but the $E 2$ decays of $0_{2}^{+}$state do not support the $\gamma$-soft rotational nature.

On the other hand, shape coexistence in the Te isotopes is still elusive [4]. The appearance of strong $\rho\left(E 0,0_{2}^{+} \rightarrow 0_{1}^{+}\right)$transition is an important spectroscopic fingerprint that mostly supports the shape coexistence in nucleus [7]. For ${ }^{124} \mathrm{Te}$, the experimental value of the electric monopole transition strength $\rho^{2}\left(E 0,0_{2}^{+} \rightarrow 0_{1}^{+}\right) \times 10^{3}$ is $12 \pm 3[8]$. This value is very similar to the corresponding transition in the $\mathrm{Cd}$ isotopes, the latter demonstrates the firm evidence for the nature of shape coexistence, leading to strong support for deformed intruder structure of the first excited $0^{+}$state in ${ }^{124} \mathrm{Te}$ isotopes, but not providing conclusive proof for shape coexistence in the ${ }^{124}$ Te yet [4]. Meanwhile, the systematic research on the known experimental information suggested that ${ }^{124}$ Te may be an example of nucleus at the $E(5)$ critical point [9]. The recent investigation indicates that ${ }^{124} \mathrm{Te}$ exhibits $E(5)$-like structure [5, 10]. But there are some debates on understanding the low-lying structure of the ${ }^{124} \mathrm{Te}($ see, e.g., Refs. [3, 10, 11] and references therein).

The $E(5)$ and $X(5)$ symmetries originally developed by Iachello [12, 13] correspond to an exact solution of the Bohr Hamiltonian for $\gamma$-independent potentials and an approximate solution of the Bohr Hamiltonian for $\gamma \approx 0^{\circ}$, respectively. From the Bohr Hamiltonian with $\gamma$ frozen at $\gamma=30^{\circ}$, a four dimensional critical point symmetry model called $Z(4)$ has been introduced [14]. Most recently, another four dimensional critical point symmetry called $T(4)$ is obtained from the Bohr Hamiltonian with the $\beta$-soft potential and for a fixed value of $\gamma$ with $0^{\circ} \leq \gamma \leq 30^{\circ}\lfloor 15]$.

In nuclear system, $E(5)$ symmetry can be used to describe a nucleus at the critical point of the second order phase transition from a spherical vibrator to a $\gamma$-soft rotor. In the interacting boson model (IBM-1) [16], which do not distinguish the proton boson and 
neutron boson, the $E(5)$ represents a nucleus that is located at the critical point of the shape phase transition between the $\mathrm{U}(5)$ and $\mathrm{O}(6)$ symmetries. Since the neutron and proton degrees of freedom are explicitly taken into account, the proton-neutron interacting boson model (IBM-2)[16] possesses a complex phase diagram [17, 18]. The critical point of second order phase transition can occur at the phase transition from $\mathrm{U}_{\pi \nu}(5)$ to $\mathrm{O}_{\pi \nu}(6)$ symmetry, and can be subsumed to the critical point of the second order phase transition in IBM-1 [17 19]. Compared with their neighboring isotopes, nuclei at or close to the critical point of shape phase transition demonstrate dramatic changes in the properties of the lowlying states, such as the energy levels, the $E 2$ transition strengths and E2 branching ratios [20]. But there is no discussion on the critical point symmetry in the real nuclei for the phase transition between $\mathrm{U}_{\pi \nu}(5)$ and $\mathrm{O}_{\pi \nu}(6)$ in the IBM-2 phase diagram, so far.

The low-lying levels of ${ }^{124} \mathrm{Te}$ have recently been examined with the $\left(n, n^{\prime}, \gamma\right)$ reaction. The spins, level energies, $B(E 2)$ transition probabilities, and multipole-mixing ratios were obtained [3]. The experiment provides us an opportunity to deeply understand the low-lying structure of ${ }^{124} \mathrm{Te}$. In this work, we study the low-lying structure of the ${ }^{124} \mathrm{Te}$ within the framework of the IBM-2, with special attention paid to the key observables of the critical point of the second order transition between $\mathrm{U}_{\pi \nu}(5)$ and $\mathrm{O}_{\pi \nu}(6)$. We also calculate the lowlying energy levels, the $E 2, M 1$ and $E 0$ transition strengths and compare the predications of the critical point symmetry of the $\mathrm{U}_{\pi \nu}(5)-\mathrm{O}_{\pi \nu}(6)$ transition with the experimental data. We attempt to describe the critical point symmetry in the ${ }^{124} \mathrm{Te}$, and reveal the low-lying structure of ${ }^{124} \mathrm{Te}$ in IBM-2 space.

The outline of the paper is as follows. In Sec. II, we introduce the Hamiltonians of IBM-2, $E 2, M 1$, and $E 0$ operators used in this work. The criteria adopted for the determination of the model parameters, and the comparison of the numerical results with experimental data and the predications of the critical point symmetry are presented in Sec. III Finally, out concluding remarks and summary are stated in Sec. IV.

\section{THEORETICAL FRAMEWORK}

The IBM-2 is an algebraic model, in which valence nucleons are coupled to form $s_{\rho}$ bosons (angular momentum $L=0$ ) and $d_{\rho}$ bosons (angular momentum $L=2$ ) with $\rho=\pi$ standing for proton bosons and $\rho=\nu$ standing for neutron bosons, respectively. The microscopic 
structure of the model suggests that only two terms are very important. One is the pairing interaction between identical nucleons, the other is the quadrupole-quadrupole interaction between non-identical nucleons. The simple standard IBM-2 Hamiltonian [16, 21] is written as

$$
\hat{H}=\varepsilon_{d \pi} \hat{n}_{d \pi}+\varepsilon_{d \nu} \hat{n}_{d \nu}+\kappa_{\pi \nu} \hat{Q}_{\pi} \cdot \hat{Q}_{\nu}
$$

where $\hat{n}_{d \rho}=d_{\rho}^{\dagger} \cdot \tilde{d}_{\rho}$ and $\hat{Q}_{\rho}=\left(s_{\rho}^{\dagger} \tilde{d}_{\rho}+d_{\rho}^{\dagger} s_{\rho}\right)^{(2)}+\chi_{\rho}\left(d_{\rho}^{\dagger} \tilde{d}_{\rho}\right)^{(2)}$ represent $d$-boson number operator and quadrupole operator, respectively. The parameter $\chi_{\rho}$ determines the type of the deformation of the quadrupole operator. $\varepsilon_{d \rho}$ is the energy of the $d$ bosons relative to the $s$ bosons, $\kappa_{\pi \nu}$ is the strength of the quadrupole-quadrupole interaction between neutron boson and proton boson.

The Hamiltonian of Eq.(1) has a much richer shape phase structure, which contains $\mathrm{U}_{\pi \nu}(5), \mathrm{O}_{\pi \nu}(6), \mathrm{SU}_{\pi \nu}(3), \overline{\mathrm{SU}_{\pi \nu}(3)}$, and $\mathrm{SU}_{\pi \nu}^{*}(3)$ dynamical symmetries, corresponding to the spherical vibrator, $\gamma$-unstable rotor, axially symmetric prolate rotor, axially symmetric oblate rotor, and triaxial rotor, respectively. The shape phase transitions in nuclei can be characterized as the quantum phase transitions in between the different dynamical symmetries in the IBM [17, 18, 22]. Although the standard IBM-2 Hamiltonian can give us more clear space of dynamical symmetry, one has to add other physical dominant interactions in order to described real nuclei more accurately. We use the following IBM-2 Hamiltonian in this paper [16, 23, 24],

$$
\hat{H}=\varepsilon_{d \pi} \hat{n}_{d \pi}+\varepsilon_{d \nu} \hat{n}_{d \nu}+\kappa_{\pi \nu} \hat{Q}_{\pi} \cdot \hat{Q}_{\nu}+\omega_{\pi \nu} \hat{L}_{\pi} \cdot \hat{L}_{\nu}+\hat{M}_{\pi \nu}
$$

where $\hat{L}_{\rho}=\sqrt{10}\left[d_{\rho}^{\dagger} \cdot \tilde{d}_{\rho}\right]^{(1)}$ is the angular momentum operator with a dipole protonneutron interaction parameter $\omega_{\pi \nu}$, and $\hat{M}_{\pi \nu}=\lambda_{2}\left(s_{\pi}^{\dagger} d_{\nu}^{\dagger}-s_{\nu}^{\dagger} d_{\pi}^{\dagger}\right)^{(2)} \cdot\left(s_{\pi} \tilde{d}_{\nu}-s_{\nu} \tilde{d}_{\pi}\right)^{(2)}$ $+\sum_{k=1,3} \lambda_{k}\left(d_{\pi}^{\dagger} d_{\nu}^{\dagger}\right)^{(k)} \cdot\left(\tilde{d}_{\pi} \tilde{d}_{\nu}\right)^{(k)}$ is the Majorana interaction, the Majorana parameters $\lambda_{k}$ $(k=1,2,3)$ represent the strength of the Majorana interaction. If one does not include the Majorana term in Eq. (2), the adopted parameters in the remaining four terms of Eq. (2) indicate that the nucleus described by the IBM-2 may locate on the the plane of $\mathrm{U}_{\pi \nu}(5)$ $\mathrm{O}_{\pi \nu}(6)-\mathrm{SU}_{\pi \nu}^{*}(3)$, because the $\hat{L}_{\pi} \cdot \hat{L}_{\nu}$ only generate the physical rotational group $\mathrm{SO}_{\pi \nu}(3)$ of the IBM-2 [16 18]. On the other hand, in Ref. 25] the authors proposed a new algebraic model $F(5)$ based on the Euclidean dynamical symmetry in five dimensions $(\mathrm{Eu}(5))$, which can build a symmetry intermediate between the $E(5)$ and $X(5)$ symmetries [22]. However, the $F(5)$ cannot directly be defined in the IBM-1 or IBM-2 due to the non-compactness of 
the $\operatorname{Eu}(5)$ group [25].

The decay properties of the low-lying states, such as the $B(E 2)$ transition probabilities, the magnetic dipole $B(M 1)$ transition strengths and the $\rho^{2}(E 0)$ values between lowest $0^{+}$ states are the signatures of phase structure. In the IBM-2, the $E 2$ transition strength is given by the following expression

$$
B\left(E 2, J^{\prime} \rightarrow J\right)=\frac{1}{2 J^{\prime}+1}\left|J\left\langle\|\hat{T}(E 2)\| J^{\prime}\right\rangle\right|^{2} .
$$

where $J^{\prime}$ and $J$ are the angular momenta for the initial and final states, respectively. The $\hat{T}(E 2)=e_{\pi} \hat{Q}_{\pi}+e_{\nu} \hat{Q}_{\nu}$ is $E 2$ operator, where the operator $Q_{\rho}$ is the same as in Eq.(11). The parameters $e_{\pi}$ and $e_{\nu}$ are the effective charges of proton bosons and neutron bosons, respectively. The values of $e_{\nu}$ and $e_{\pi}$ could be taken differently.

In the proton-neutron interacting boson model, the magnetic dipole $M 1$ transition operator is defined as

$$
\hat{T}(M 1)=\sqrt{\frac{3}{4 \pi}}\left(g_{\nu} \hat{L}_{\nu}+g_{\pi} \hat{L}_{\pi}\right),
$$

where the $\hat{L}_{\rho}$ is the same as in Eq.(2). The $g_{\pi}$ and $g_{\nu}$ are the effective proton and neutron boson $g$-factors, repectively. Typically, one can take the values of $g_{\pi}=1, g_{\nu}=0$ in the calculations [21].

The $E 0$ transition matrix element $\rho$ in the IBM-2 is written as [26, 27]

$$
\rho\left(E 0, J^{\prime} \rightarrow J\right)=\frac{Z}{e R^{2}}\left[\beta_{0 \pi}\left\langle J\left\|\hat{T}_{\pi}(E 0)\right\| J^{\prime}\right\rangle+\beta_{0 \nu}\left\langle J\left\|\hat{T}_{\nu}(E 0)\right\| J^{\prime}\right\rangle\right],
$$

where $R=1.2 A^{1 / 3} \mathrm{fm}, \beta_{0 \pi}$, and $\beta_{0 \nu}$ are the effective monopole charges of proton and neutron boson in units of $\mathrm{efm}^{2}$, respectively. The $E 0$ operator is expressed as $\hat{T}(E 0)=\beta_{0 \pi} \hat{T}_{\pi}(E 0)+$ $\beta_{0 \nu} \hat{T}_{\nu}(E 0)=\beta_{0 \pi} \hat{n}_{d \pi}+\beta_{0 \nu} \hat{n}_{d \nu}$, where the $\hat{n}_{d \rho}$ is the same as in Eq.(1).

\section{RESULTS AND DISCUSSION}

The ${ }^{124} \mathrm{Te}$ situates at the position between vibrational nucleus and deformed nucleus [28]. The IBM provides a powerful tool to describe the nuclear shapes and the shape phase transitions. With this approach, a lot of works have been devoted to describe the properties of the low-lying states of ${ }^{124} \mathrm{Te}$ isotope. In IBM-1, the early work regarded the ${ }^{124} \mathrm{Te}$ as an example of $\mathrm{O}(6)$ symmetry, but the energy levels of yrast states of ${ }^{124} \mathrm{Te}$ differ from the $\mathrm{O}$ (6) limit [29]. The further systematic investigations found that ${ }^{124} \mathrm{Te}$ was close to the 
U(5) symmetry [30, 31]. Moreover, recent results of experiments and calculations indicated that ${ }^{124}$ Te may posses the $E(5)$ features [3, 5, 9, 10]. In IBM-2, the calculations with and without mixed configuration suggested that ${ }^{124}$ Te is an $\mathrm{O}(6)$-like nucleus, but the $B(E 2)$ decay pattern is not consistent with this symmetry [32]. The subsequent study shows that neither $\mathrm{O}(6)$ nor $\mathrm{U}(5)$ symmetries can describe the ${ }^{124}$ Te well [33], although the potentialenergy surface of ${ }^{124} \mathrm{Te}$ exhibits an obvious manifestation of $\mathrm{O}(6)$ limit [34]. Another recent experiment and IBM calculation support that ${ }^{124} \mathrm{Te}$ shows the soft triaxial behavior [6]. Although the debate on the low-lying structure of the ${ }^{124} \mathrm{Te}$ still exists, it is indubitable that all these investigations indicated that ${ }^{124} \mathrm{Te}$ is a transitional nucleus between vibration $\mathrm{U}(5)$ symmetry and $\gamma$-unstable $\mathrm{O}(6)$ symmetry in IBM, and might accompany with somewhat soft triaxial rotation.

To describe the low-lying structure of ${ }^{124} \mathrm{Te}$, we select the doubly closed shell ${ }_{50}^{132} \mathrm{Sn}_{82}$ as the inert core. There are $N_{\pi}=1$ particle-like boson beyond the $Z=50$ major shell and $N_{\nu}=5$ hole-like neutron bosons from the $N=82$ shell closure, and the total number of bosons is $N_{B}=6$. Considering that the proton boson is particle-like and the neutron bosons are hole-like based on the different single particle orbitals in ${ }^{124} \mathrm{Te}$, we take different relative energies for $d$ neutron and proton boson in this case, i.e., $\varepsilon_{d \pi} \neq \varepsilon_{d \nu}$ just as in Refs.[35 37]. To describe the different type of valence neutrons and protons exhibiting opposite intrinsic quadrupole deformation, and obtain the $\gamma$-unstable $\mathrm{O}(6)$ symmetry, we adopt the $\chi_{\pi}+\chi_{\nu}=0$. In order to introduce the somewhat character of soft triaxial rotation in ${ }^{124} \mathrm{Te}$, the value of $\chi_{\pi}$ and $\chi_{\nu}$ should not be zero. In principle, the parameter $\varepsilon_{d \rho}$ mainly leads to vibrational solutions, while $\kappa_{\pi \nu}$ drives the system into a deformed shape, and the description of the electromagnetic properties is very sensitive to the parameters $\chi_{\rho}$. The dipole interaction $\hat{L}_{\pi} \cdot \hat{L}_{\nu}$ can improve the rotational spectrum and can adjust the position of the $4_{1}^{+}$relative to $2_{2}^{+}$state, but it does not affect the wave vectors [38]. The Majoranan operator mainly contributes to the mixed symmetry states including the scissors mode. All the free parameters in our calculations were fixed to reproduce the experimental energies and electromagnetic transition strengths of ${ }^{124} \mathrm{Te}$. By fitting to the energy levels of experimental data especially for the $2_{1}^{+}$state, we obtain the parameters of IBM-2 in this work as follows: $\varepsilon_{d \pi}=1.220 \mathrm{MeV}, \varepsilon_{d \nu}=0.710 \mathrm{MeV}, \kappa_{\pi \nu}=-0.140 \mathrm{MeV}, \chi_{\pi}=-\chi_{\nu}=-1.00$, $\omega_{\pi \nu}=-0.055 \mathrm{MeV}, \lambda_{1}=-0.700 \mathrm{MeV}, \lambda_{2}=0.220 \mathrm{MeV}$, and $\lambda_{3}=-0.100 \mathrm{MeV}$. The IBM-2 Hamiltonian will be numerically diagonalized by using the NPBOS code [39]. In Figure 1, we 


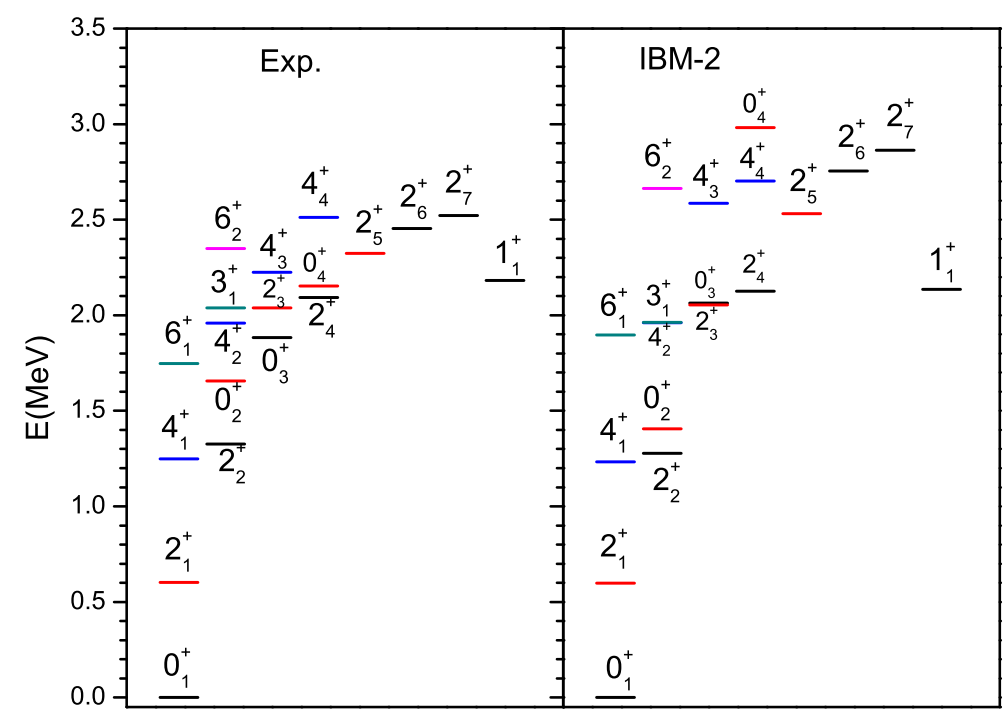

FIG. 1: Comparison of the calculated and experimental [3, 40] low-lying positive-parity levels of ${ }^{124} \mathrm{Te}$.

compare the calculated level energies with the experimental results [3, 40]. The experimental level energies in Figure 1 are separated into different bands based only on their order of appearance, where only the low-spin positive parity states with uniquely assigned spin and parity are displayed.

Figure 1 shows that the theoretical levels are good consistent with the corresponding experimental ones. In particular, the $6_{1}^{+}$state is dominated by two valence protons and is relatively unaffected by the increase of neutrons, the $6_{1}^{+}$states remain at a nearly constant excitation energy for even-even ${ }^{122-130}$ Te isotopes [3]. By introducing the dipole interaction term into the Hamiltonian [41 43], the structure of the yrast bands including the $6_{1}^{+}$level are reproduced by the theoretical predications. Similar to the $6_{1}^{+}$state, the observed energy of $4_{2}^{+}$level is only changed about $135 \mathrm{keV}$ from ${ }^{122} \mathrm{Te}$ to ${ }^{130} \mathrm{Te}$ isotopes. The present calculation reproduces the experimental energy of the $4_{2}^{+}$level nicely. Meanwhile, the present calculated energies of the $2_{3,4,5}^{+}$, and $6_{2}^{+}$levels agree with the experimental observations. Even though the $0_{2}^{+}$level is obviously higher than that is expected for a two-phonon multiple state, the description of the first two excitation $0^{+}$states in energy sequence is satisfactory. 
Furthermore, the calculated energy level of the first scissor mode $1_{1}^{+}$perfectly reproduces the experimental data. However, there are some discrepancies between the experimental observations and the theoretical predications for higher energy states, such as $2_{6}^{+}$, and $2_{7}^{+}$ states, which is a general feature of this model [47]. For the $0_{4}^{+}$state, the calculated level is significantly higher than the experimental one. The IBM-2 with the mixed configuration shows that $0_{4}^{+}$is an intruder state in ${ }^{124} \mathrm{Te}$ and locates outside the IBM-2 space [32].

In seeking to investigate the properties of the $E 2$ transitions in ${ }^{124} \mathrm{Te}$, the effective charges of proton and neutron bosons were determined to reproduce the experimental $B(E 2)$ values. Using the same manner as in Refs. [48, 49] and exactly fitting to the experimental data of $B\left(E 2,2_{1}^{+} \rightarrow 0_{1}^{+}\right)=31(5)$ W.u., we obtain $e_{\pi}=3.780 \sqrt{\text { W.u. }}, e_{\nu}=1.670 \sqrt{\text { W.u. }}$ The theoretical calculation of $B(E 2)$ values for ${ }^{124} \mathrm{Te}$ in comparison with the available experimental data [1, 3, 10, 44 46] is given in Table I.

Table 【 shows that the computed $B(E 2)$ transition strengths are in overall agreement with the experimental data, although most cases of the experimental value have a large uncertainty. In more detail, the typical strongly collective $E 2$ transitions with tens of Weisskopf units are described by the theoretical predications very well, some of them consist with each other within the experimental uncertainty. It is noteworthy that the experimental $B\left(E 2,4_{1}^{+} \rightarrow 2_{1}^{+}\right)$transition strength is $35.9( \pm 17)$ W.u. in Ref. [3], which is taken from Ref.[6]. In Ref.[45], the experimental values show that the lower and upper limits of this transition probability are 27.3 and 163.5 W.u., respectively. Similar to Ref.[44], we adopt the experimental averaged value $54.51 \mathrm{~W} . u$. for this transition, the detail can be seen in the Ref. [50], one can find that the experimental value agrees with the present calculated value. At the same time, the experimental relatively strong $B(E 2)$ transitions, which can be comparable to the experimental transition probability of the first excited state $2_{1}^{+}$decay to the ground state with dozens of W.u., are reproduced by the calculated results perfectly except the $B\left(E 2,4_{3}^{+} \rightarrow 2_{2}^{+}\right)$transition strength. Furthermore, the calculated results are in good description of the properties of the experimental weakly collective $E 2$ transitions with about one or even less than one W.u.. The theoretical E2 transition strengths from the scissor mode $1_{1}^{+}$to the $2_{1}^{+}$and $2_{2}^{+}$states are in agreement with the corresponding experimental data, although the theoretical values are a little higher than the experimental data.

To identify where the ${ }^{124} \mathrm{Te}$ can be placed in the $\mathrm{U}_{\pi \nu}(5)$ to $\mathrm{O}_{\pi \nu}(6)$ transition, we focus on a set of key observables [51], such as the energy ratios $R_{4_{1} / 2_{1}}=E\left(4_{1}^{+}\right) / E\left(2_{1}^{+}\right), R_{2_{2} / 2_{1}}=$ 
TABLE I: Experimental E2 transition strengths in ${ }^{124}$ Te in units of W.u. are compared with the calculated results. The experimental data are taken from Refs. [1, 3, 10, 44 46]

\begin{tabular}{|c|c|c|}
\hline$J_{i}^{\pi} \rightarrow J_{f}^{\pi}$ & Expt. & IBM-2 \\
\hline $2_{1}^{+} \rightarrow 0_{1}^{+}$ & $31_{5}^{5}$ & 31.02 \\
\hline $4_{1}^{+} \rightarrow 2_{1}^{+}$ & 54.51 & 48.06 \\
\hline $2_{2}^{+} \rightarrow 2_{1}^{+}$ & $55.5_{99}^{109}$ & 49.53 \\
\hline $2_{2}^{+} \rightarrow 0_{1}^{+}$ & $0.83_{16}^{23}$ & 0.24 \\
\hline $0_{2}^{+} \rightarrow 2_{1}^{+}$ & $20_{4}^{4}$ & 27.92 \\
\hline $0_{3}^{+} \rightarrow 2_{2}^{+}$ & 50.00 & 48.51 \\
\hline $4_{2}^{+} \rightarrow 2_{2}^{+}$ & $14.1_{28}^{30}$ & 29.94 \\
\hline $4_{2}^{+} \rightarrow 4_{1}^{+}$ & $12.7_{59}^{74}$ & 27.06 \\
\hline $4_{2}^{+} \rightarrow 2_{1}^{+}$ & $4.3_{9}^{9}$ & 0.31 \\
\hline $3_{1}^{+} \rightarrow 2_{2}^{+}$ & $59_{10}^{10}$ & 42.65 \\
\hline $2_{3}^{+} \rightarrow 0_{1}^{+}$ & $0.26_{4}^{4}$ & 2.08 \\
\hline $2_{3}^{+} \rightarrow 2_{1}^{+}$ & $0.025_{3}^{4}$ & 0.50 \\
\hline $2_{3}^{+} \rightarrow 2_{2}^{+}$ & $\leq 2.7_{15}^{19}$ & 4.03 \\
\hline $2_{3}^{+} \rightarrow 4_{1}^{+}$ & $0.81_{81}^{104}$ & 5.38 \\
\hline $2_{3}^{+} \rightarrow 3_{1}^{+}$ & $0.26_{4}^{5}$ & 0.37 \\
\hline $2_{4}^{+} \rightarrow 2_{2}^{+}$ & $0.29_{33}^{39}$ & 1.85 \\
\hline $2_{4}^{+} \rightarrow 2_{1}^{+}$ & $1.6_{3}^{3}$ & 1.39 \\
\hline $2_{4}^{+} \rightarrow 0_{1}^{+}$ & $0.053_{14}^{17}$ & 2.16 \\
\hline $0_{4}^{+} \rightarrow 2_{1}^{+}$ & $<0.5$ & 0.04 \\
\hline $0_{4}^{+} \rightarrow 2_{2}^{+}$ & $<50$ & 0.03 \\
\hline $1_{1}^{+} \rightarrow 2_{1}^{+}$ & $1.2_{3}^{4}$ & 4.99 \\
\hline $1_{1}^{+} \rightarrow 2_{2}^{+}$ & $0.74_{38}^{38}$ & 1.13 \\
\hline $4_{3}^{+} \rightarrow 2_{2}^{+}$ & $13.4_{39}^{49}$ & 1.16 \\
\hline $4_{3}^{+} \rightarrow 4_{1}^{+}$ & $6.7_{20}^{23}$ & 2.89 \\
\hline $4_{3}^{+} \rightarrow 2_{1}^{+}$ & $1.9_{5}^{6}$ & 0.95 \\
\hline $2_{5}^{+} \rightarrow 2_{2}^{+}$ & $0.61_{30}^{40}$ & 1.02 \\
\hline $2_{5}^{+} \rightarrow 2_{1}^{+}$ & $0.06_{6}^{7}$ & 0.15 \\
\hline $2_{5}^{+} \rightarrow 0_{1}^{+}$ & $0.078_{42}^{50}$ & 0.09 \\
\hline
\end{tabular}


Table I(continued)

\begin{tabular}{ccc}
\hline$J_{i}^{\pi} \rightarrow J_{f}^{\pi}$ & Expt. & IBM-2 \\
\hline $2_{6}^{+} \rightarrow 2_{2}^{+}$ & $1.6_{5}^{5}$ & 1.67 \\
$2_{6}^{+} \rightarrow 4_{1}^{+}$ & $1.7_{4}^{5}$ & 0.00 \\
$2_{6}^{+} \rightarrow 2_{1}^{+}$ & $0.046_{6}^{8}$ & 0.23 \\
$2_{6}^{+} \rightarrow 0_{1}^{+}$ & $0.12_{2}^{3}$ & 0.01 \\
$4_{4}^{+} \rightarrow 4_{1}^{+}$ & $2.6_{7}^{9}$ & 0.10 \\
$4_{4}^{+} \rightarrow 2_{1}^{+}$ & $0.051_{22}^{28}$ & 0.72 \\
$2_{7}^{+} \rightarrow 2_{1}^{+}$ & $4.0_{4}^{4}$ & 0.56 \\
$2_{7}^{+} \rightarrow 2_{2}^{+}$ & $0.20_{11}^{10}$ & 0.00 \\
$2_{7}^{+} \rightarrow 4_{1}^{+}$ & $<1.6$ & 1.10 \\
\hline \hline
\end{tabular}

$E\left(2_{2}^{+}\right) / E\left(2_{1}^{+}\right), R_{0_{2} / 2_{1}}=E\left(0_{2}^{+}\right) / E\left(2_{1}^{+}\right), R_{0_{3} / 0_{2}}=E\left(0_{3}^{+}\right) / E\left(0_{2}^{+}\right)$, and the $B(E 2)$ ratios $R_{B, 42}=$ $B\left(E 2,4_{1}^{+} \rightarrow 2_{1}^{+}\right) / B\left(E 2,2_{1}^{+} \rightarrow 0_{1}^{+}\right), R_{B, 22}=B\left(E 2,2_{2}^{+} \rightarrow 2_{1}^{+}\right) / B\left(E 2,2_{1}^{+} \rightarrow 0_{1}^{+}\right)$and $R_{B, 02}=$ $B\left(E 2,0_{2}^{+} \rightarrow 2_{1}^{+}\right) / B\left(E 2,2_{1}^{+} \rightarrow 0_{1}^{+}\right)$, which reflect the characteristics of the nucleus behavior at critical point of the phase transition from $\mathrm{U}_{\pi \nu}(5)$ to $\mathrm{O}_{\pi \nu}(6)$ in IBM-2 space [17], and are the most crucial nuclear structure indicators [52]. Some of the indicators can even distinguish the first order quantum phase transition from the second order quantum phase transition [53 55]. The values of these key observables at the critical point of the phase transition from $\mathrm{U}_{\pi \nu}(5)$ to $\mathrm{O}_{\pi \nu}(6)$ for the infinite numbers of bosons [12], as well as the experimental data and the calculated results in ${ }^{124} \mathrm{Te}$ are listed in Table II for comparison.

Table II shows that the overall agreement is well. On the energy ratios, the experimental ratio $R_{2_{2} / 2_{1}}$ is almost exactly reproduced to the the critical point of the $\mathrm{U}_{\pi \nu}(5)-\mathrm{O}_{\pi \nu}(6)$ transition. The experimental ratio $R_{0_{3} / 0_{2}}$ is very close to the value of CPST. Meanwhile, the experimental energy of the $0_{2}^{+}$state relative to $E\left(2_{1}^{+}\right)$satisfactorily matches the predicated value of CPST. The experimental $R_{4_{1} / 2_{1}}$ lies between $\mathrm{U}_{\pi \nu}(5)\left(R_{2_{2} / 2_{1}}=2.00\right)$ and $\mathrm{O}_{\pi \nu}(6)$ $\left(R_{2_{2} / 2_{1}}=2.50\right)$ limits, although it deviates slightly from the predicated one. On the $B(E 2)$ ratios, the experimental $R_{B, 42}$ is well reproduced by the theoretical predication and clearly points to a structure intermediate between $\mathrm{U}_{\pi \nu}(5)\left(R_{B, 42}=2.00\right)$ and $\mathrm{O}_{\pi \nu}(6)\left(R_{B, 42}=10 / 7\right)$. Meanwhile, the experimental value of $R_{B, 22}$ approaches the predicted value of the critical point theory, too. In particular, the information of $R_{B, 02}$ is a signature for identifying 
TABLE II: Comparison of the key observables of the states at the critical point of the $\mathrm{U}_{\pi \nu}(5)-$ $\mathrm{O}_{\pi \nu}(6)$ transition (taken from Ref.[12] and denoted as CPST), the experimental data of ${ }^{124} \mathrm{Te}$ (taken from Refs. [1, 3, 44 46] and labeled as ${ }^{124} \mathrm{Te}$ ) and the calculated results (labeled as IBM-2).

\begin{tabular}{llll}
\hline & CPST & ${ }^{124} \mathrm{Te}$ & IBM-2 \\
\hline$R_{4_{1} / 2_{1}}$ & 2.20 & 2.07 & 2.06 \\
$R_{2_{2} / 2_{1}}$ & 2.20 & 2.20 & 2.13 \\
$R_{0_{2} / 2_{1}}$ & 3.03 & 2.75 & 2.35 \\
$R_{0_{3} / 0_{2}}$ & 1.18 & 1.14 & 1.46 \\
$R_{B, 42}$ & 1.68 & 1.74 & 1.55 \\
$R_{B, 22}$ & 1.68 & 1.79 & 1.60 \\
$R_{B, 02}$ & 0.86 & 0.65 & 0.90 \\
\hline \hline
\end{tabular}

the $\mathrm{U}_{\pi \nu}(5)$ from the $\mathrm{O}_{\pi \nu}(6)$ symmetry. In $\mathrm{U}_{\pi \nu}(5)$ limit the $B\left(E 2,0_{2}^{+} \rightarrow 2_{1}^{+}\right)$transition strength is 2 times larger than $\left(B\left(E 2,2_{1}^{+} \rightarrow 0_{1}^{+}\right)\right.$. In $\mathrm{O}_{\pi \nu}(6)$ limit the $B\left(E 2,0_{2}^{+} \rightarrow 2_{1}^{+}\right)$ transition is forbidden [56]. Here, both the experimental and theoretical values of the $R_{B, 02}$ can correctly reflect the nature of the $B\left(E 2,0_{2}^{+} \rightarrow 2_{1}^{+}\right)$transition, although the calculated value overestimates about 1.5 times than experimental one. Therefore, all the available experimental information on the key observables for ${ }^{124} \mathrm{Te}$ is in good agreement with the predictions of critical point of the $\mathrm{U}_{\pi \nu}(5)-\mathrm{O}_{\pi \nu}(6)$ transition. Meanwhile, Table I also shows that the present calculation of the characteristic feature of ${ }^{124} \mathrm{Te}$ is remarkable. However, the $R_{0_{2} / 2_{1}}$ of CPST seems larger than the IBM's in TableII, the reason is that the calculation of CPST is given for the infinite $-N_{B}$ limit [12, 51]. For the finite number of bosons, Ref.[17] gives that the $R_{0_{2} / 2_{1}}$ of a nucleus at the critical point of the phase transition from $\mathrm{U}_{\pi \nu}(5)$ to $\mathrm{O}_{\pi \nu}(6)$ with $N_{\pi}=N_{\nu}=5$ is 2.48 , which agrees the result of present calculation. From the above discussion, we conclude that the ${ }^{124} \mathrm{Te}$ may be a nucleus at the critical point of the $\mathrm{U}_{\pi \nu}(5)-\mathrm{O}_{\pi \nu}(6)$ transition. Consequently, the $0_{2}^{+}$state is the possible lowest state of the first excited family of intrinsic levels predicted by the critical point symmetry.

An advantage of IBM-2 over IBM-1 is that the former can study the influence of the critical point symmetry on magnetic dipole transitions between the low-lying states [23]. In IBM-2, the pure $\mathrm{O}_{\pi \nu}(6)$ symmetry can be obtained only with $\chi_{\pi}=\chi_{\nu}=0$. In this case, 
there does not exist any asymmetry between $\chi_{\pi}$ and $\chi_{\nu}$. It means that the mixed symmetry components could not mix into the low-lying states. Therefore, the $M 1$ transitions between these states are forbidden in $\mathrm{O}_{\pi \nu}(6)$ limit [21]. The theoretical predictions show that the $B(M 1)$ transition strengths among the low-lying states almost vanish, when a nucleus is at the critical point of the $\mathrm{U}_{\pi \nu}(5)-\mathrm{O}_{\pi \nu}(6)$ transition [17]. For the scissors mode state $1_{1}^{+}$, the $B\left(M 1,1_{1}^{+} \rightarrow 0_{1}^{+}\right)$transition strength is $3 N_{\pi} N_{\nu}\left(g_{\nu}-g_{\pi}\right)^{2} / 4 \pi\left(2 N_{B}+1\right)$ for $\mathrm{O}_{\pi \nu}(6)$ limit, but it is forbidden for $\mathrm{U}_{\pi \nu}(5)$ limit. The $B\left(M 1,1_{1}^{+} \rightarrow 0_{2}^{+}\right)$value is $3 N_{\pi} N_{\nu}\left(g_{\nu}-g_{\pi}\right)^{2} / \pi N_{B}\left(N_{B}-1\right)$ for $\mathrm{U}_{\pi \nu}(5)$ limit, whereas vanish for $\mathrm{O}_{\pi \nu}(6)$ limit $[23,57,58]$. At the critical point of $\mathrm{U}_{\pi \nu}(5)^{-}$ $\mathrm{O}_{\pi \nu}(6)$ transition, the $1_{1}^{+}$state has an allowed $M 1$ decays to first and second $0^{+}$states. In the case of ${ }^{124} \mathrm{Te}$, the $B\left(M 1,1_{1}^{+} \rightarrow 0_{1}^{+}\right)$is $0.10 \mu_{N}^{2}$ for $\mathrm{O}_{\pi \nu}(6)$ limit, and $B\left(M 1,1_{1}^{+} \rightarrow 0_{2}^{+}\right)$ is $0.50 \mu_{N}^{2}$ for $\mathrm{U}_{\pi \nu}(5)$ limit with the typical values $g_{\pi}=1, g_{\nu}=0$. Indeed, only a few $M 1$ transitions with very small absolute values of $B(M 1)$ among the low-lying states in ${ }^{124} \mathrm{Te}$ have been measured [46, 59]. By using the Eq.(44) and also taking $g_{\pi}=1$ and $g_{\nu}=0$, the calculated $M 1$ transition probabilities, as well as the corresponding available experimental data for the scissors mode and the low-lying states in ${ }^{124} \mathrm{Te}$ are given in Table 【II.

As can be seen from Table 【II, all the experimental $B(M 1)$ transition strengths between the lowest $2^{+}$states are of the order of about $0.01 \mu_{N}^{2}$ or even less, which is far from the typical $B(M 1)$ value of the mixed symmetry state. This demonstrates that the observed $M 1$ transitions are in qualitative agreement with the predictions of the critical point symmetry of the $\mathrm{U}_{\pi \nu}(5)-\mathrm{O}_{\pi \nu}(6)$ transition. At the same time, Table III shows that the calculated results reproduce the characteristics of the $M 1$ transitions among low-lying states very fairly. In particular, the calculated $B\left(M 1,1_{1}^{+} \rightarrow 0_{1}^{+}\right)$strength is close to the experimental one, which is comparable the predicted value of the critical point between the $\mathrm{U}_{\pi \nu}(5)$ and $\mathrm{O}_{\pi \nu}(6)$ limits. This indicates that both the experimental and the calculated results of the $B\left(M 1,1_{1}^{+} \rightarrow 0_{1}^{+}\right)$transition reflect the property of the nucleus at the critical point of the $\mathrm{U}_{\pi \nu}(5)-\mathrm{O}_{\pi \nu}(6)$ transition. Unfortunately, the $B\left(M 1,1_{1}^{+} \rightarrow 0_{2}^{+}\right)$strength in ${ }^{124} \mathrm{Te}$ has not been measured, the calculated value for this transition is $0.035 \mu_{N}^{2}$, locating between the $\mathrm{U}_{\pi \nu}(5)$ and $\mathrm{O}_{\pi \nu}(6)$ transition similar to the case of $B\left(M 1,1_{1}^{+} \rightarrow 0_{1}^{+}\right)$transition. All these results indicate that both the calculated and experimental $M 1$ transitions are consistent with the characteristic behavior of the ${ }^{124} \mathrm{Te}$ nucleus at the critical point of the $\mathrm{U}_{\pi \nu}(5)-\mathrm{O}_{\pi \nu}(6)$ transition.

However, some deviations between the theoretical and experimental $M 1$ transition 
TABLE III: Comparison of the calculated $M 1$ transition strengths (in units of $\mu_{N}^{2}$ ) and the experimental data for ${ }^{124} \mathrm{Te}$. The experimental data are taken from Refs. [11, 45, 46, 60].

\begin{tabular}{lll}
\hline Transition & Expt. & IBM-2 \\
\hline $2_{2}^{+} \rightarrow 2_{1}^{+}$ & 0.0003 & 0.006 \\
$2_{3}^{+} \rightarrow 2_{2}^{+}$ & 0.004 & 0.025 \\
$2_{3}^{+} \rightarrow 2_{1}^{+}$ & 0.013 & 0.072 \\
$2_{4}^{+} \rightarrow 2_{2}^{+}$ & 0.001 & 0.031 \\
$2_{4}^{+} \rightarrow 2_{1}^{+}$ & 0.004 & 0.075 \\
$1_{1}^{+} \rightarrow 0_{1}^{+}$ & 0.04 & 0.029 \\
$1_{1}^{+} \rightarrow 0_{2}^{+}$ & & 0.034 \\
\hline \hline
\end{tabular}

strengths also are displayed in Table III, all the calculated $M 1$ values are a little larger than the corresponding experimental data. In IBM-2, $\chi_{\pi}=-\chi_{\nu} \neq 0$ leads to spectrum having many of the $\mathrm{O}(6)$ features. In addition, some mixed symmetry components from the asymmetry of $\chi_{\pi}$ and $\chi_{\nu}$ mix into the low-lying states beside the pure mixed symmetry states at higher energies [21, 61]. On the other hand, $\chi_{\pi}=-\chi_{\nu} \neq 0$ makes the nucleus have some features of the $\mathrm{SU}_{\pi \nu}^{*}(3)$ symmetry, because the proton and neutron have opposite types of deformation [18]. The calculated $B(M 1)$ values among the lowest states are systematically slightly larger than the experimental data, which implies that the description of ${ }^{124} \mathrm{Te}$ with the character of the $\mathrm{SU}_{\pi \nu}^{*}(3)$ is slightly rather than the actual nucleus. Furthermore, the small $B(M 1)$ values among the low-lying states in ${ }^{124} \mathrm{Te}$ also indicate that ${ }^{124} \mathrm{Te}$ may contain some components of $\mathrm{SU}_{\pi \nu}^{*}(3)$ symmetry. Therefore, the ${ }^{124} \mathrm{Te}$ may be the nucleus at the critical point of the $\mathrm{U}_{\pi \nu}(5)-\mathrm{O}_{\pi \nu}(6)$ transition and with somewhat $\mathrm{SU}_{\pi \nu}^{*}(3)$ symmetry.

The electric monopole transition strengths between $0^{+}$states can be considered as a signature of shape coexistence [7], quantum phase transitions [62], and can reflect the properties of $E(5)$ dynamical symmetry [9]. In fact, the $\rho^{2}\left(E 0,0_{2}^{+} \rightarrow 0_{1}^{+}\right)$value of ${ }^{124} \mathrm{Te}$ has been measured [8]. But the $\rho^{2}(E 0)$ value on the first excited $0^{+}$state in ${ }^{124} \mathrm{Te}$ is a puzzle for a long time as mentioned in the introduction [4]. In IBM, the E0 transitions do not occur in the $\mathrm{U}(5)$ dynamical limit, because $E 0$ operator is proportional to $\hat{n}_{d}$ [63]. While in $\mathrm{O}(6)$ limit, the selection rules require the $E 0$ transition from the $0_{2}^{+}$to ground state is forbidden [64]. 
For the predicted $E(5)$ symmetry, the $0_{2}^{+}$level, which is the lowest member (zero phonon) of the first excited family, should have an allowed $E 0$ branch to the $0_{1}^{+}$state. However, so far the studies of the behavior of $E(5)$ symmetry in ${ }^{124} \mathrm{Te}$ have not provide a detailed analysis of the properties of $E 0$ transitions. In IBM-2 space, all the symmetrical states have correspondence with the IBM-1 states, therefore, IBM-2 subsumes the critical point of second order transition of IBM-1 [17, 23]. The critical point symmetry of $\mathrm{U}_{\pi \nu}(5)-\mathrm{O}_{\pi \nu}(6)$ bears considerably resemblance to the predictions of the $E(5)$ symmetry [17]. Subsequently, the $0_{2}^{+}$level should also have an allowed $E 0$ decay to the ground state in this prediction of the critical point symmetry. We take the values of parameters $\beta_{0 \nu}$ and $\beta_{0 \pi}$ as in Ref. [65], namely, $\beta_{0 \nu}=0.25$ and $\beta_{0 \pi}=0.10 \mathrm{efm}^{2}$. The calculated $\rho^{2}\left(E 0,0_{2}^{+} \rightarrow 0_{1}^{+}\right) \times 10^{3}$ value is 11.00, which is consistent with the experimental data within the experimental uncertainty. The theoretical and experimental $E 0$ transitions prove that the $0_{2}^{+}$state may be interpreted as the lowest state of the first excited family of intrinsic levels in the critical point symmetry of second order $\mathrm{U}_{\pi \nu}(5)-\mathrm{O}_{\pi \nu}(6)$ transition.

It is well known that the low-lying yrast states in other Te isotopes such as ${ }^{112,114} \mathrm{Te}$ show the similar behavior, i.e., typical vibrational like spectra but the other properties deviate from vibrator [6, 66 68]. All these Te isotopes locate around the midshell ${ }^{118} \mathrm{Te}$. The large-scale shell-model calculations indicate that the behavior of the $B(E 2)$ values of these nuclei is related to the competition between the seniority coupling and the neutron-proton correlations. From the point of IBM model, ${ }^{112} \mathrm{Te}$ is below the midshell ${ }^{118} \mathrm{Te}$, it has $N_{\nu}=5$ bosons beyond $N=50$ major shell. Both ${ }^{112} \mathrm{Te}$ and ${ }^{124} \mathrm{Te}$ have the same numbers of bosons with $N_{\pi}=1$ and $N_{\nu}=5$, therefore, they show the similar properties of spectrum. As for ${ }^{114} \mathrm{Te}$, the measured ratio of $B\left(E 2,4_{1}^{+} \rightarrow 2_{1}^{+}\right)$to $B\left(E 2,2_{1}^{+} \rightarrow 0_{1}^{+}\right)$is smaller than one, which is very unusual deformation, no theoretical models can give a satisfied description [68].

\section{CONCLUSION}

In summary, the low-lying structure of the ${ }^{124} \mathrm{Te}$ has been investigated within the framework of IBM-2. The calculated low-lying energy levels are good consistent with the corresponding experimental data. In particular, the $6_{1}^{+}, 4_{2}^{+}$levels, which are remaining at a nearly constant excitation energy from ${ }^{122} \mathrm{Te}$ to ${ }^{130} \mathrm{Te}$ isotopes, have been reproduced by calculated results very satisfactorily. Meanwhile, the description of the first two excitated $0^{+}$states in 
energy sequence is very well, although the $0_{2}^{+}$level is obviously higher than that is expected for a two-phonon multiple state. Furthermore, the calculated energy level of the first scissor mode $1_{1}^{+}$perfectly reproduces the experimental data.

The calculations show that the computed $B(E 2)$ transition strengths are in the overall agreement with the experimental data. The observed typical strongly collective $E 2$ transitions with tens of Weisskopf units, and the most of the experimental relatively strong $B(E 2)$ transitions comparable to the experimental $B\left(E 2,2_{1}^{+} \rightarrow 0_{1}^{+}\right)$transition probability with dozens of W.u., are reproduced by the theoretical predications nicely. Furthermore, the calculated results provide the perfect description of the properties of the experimentally weakly collective E2 transition with about one or even less than one W.u.. Particularly, the theoretical $E 2$ transitions from the scissor mode $1_{1}^{+}$to the $2_{1}^{+}$and $2_{2}^{+}$states are in good agreement with the corresponding experimental data, although the theoretical values are slightly higher than the experimental data.

By comparing the key observables of the states at the critical point of the $\mathrm{U}_{\pi \nu}(5)-\mathrm{O}_{\pi \nu}(6)$ transition with the experimental data and IBM-2 calculations, we show that both the experimental data and the theoretical results of the ${ }^{124} \mathrm{Te}$ agree with the predicted properties of the states at the critical point of the phase transition from $\mathrm{U}_{\pi \nu}(5)$ to $\mathrm{O}_{\pi \nu}(6)$ very well. All these information on the key observables indicates that the ${ }^{124} \mathrm{Te}$ may be a nucleus at the critical point of the $\mathrm{U}_{\pi \nu}(5)-\mathrm{O}_{\pi \nu}(6)$ transition. Consequently, the $0_{2}^{+}$state is the possible lowest state of the first excited family of intrinsic levels predicted by the critical point symmetry.

At the same time, the characteristics of the $B(M 1)$ transition strengths among the lowlying states are consistent with the predications of the critical point of the transition from $\mathrm{U}_{\pi \nu}(5)$ to $\mathrm{O}_{\pi \nu}(6)$ well, and the overall agreement between the experiment and the theory for characteristics of the $B(M 1)$ transition strengths is perfect. Especially, both the experimental and theoretical $B\left(M 1,1_{1}^{+} \rightarrow 0_{1}^{+}\right)$values are located between the $\mathrm{U}_{\pi \nu}(5)$ and $\mathrm{O}_{\pi \nu}(6)$ limits. By analyzing the calculated results and the structure parameters of quadrupole operator in this work, all these quantities support that the ${ }^{124}$ Te may be the nucleus at the critical point of the $\mathrm{U}_{\pi \nu}(5)-\mathrm{O}_{\pi \nu}(6)$ transition and with somewhat feature of $\mathrm{SU}_{\pi \nu}^{*}(3)$ symmetry.

The calculated $\rho^{2}\left(E 0,0_{2}^{+} \rightarrow 0_{1}^{+}\right) \times 10^{3}$ value also reproduced exactly the observed data. Both the theoretical and experimental properties of $E 0$ transition indicate that the $0_{2}^{+}$state 
in ${ }^{124}$ Te may be interpreted as the lowest state of the first excited family of intrinsic levels at the critical point of second order $\mathrm{U}_{\pi \nu}(5)-\mathrm{O}_{\pi \nu}(6)$ transition. Therefore, we conclude that the ${ }^{124} \mathrm{Te}$ is a possible nucleus at the critical point of the second order phase transition from vibration to unstable rotation, and such a critical point contains somewhat triaxial rotation. The $0_{2}^{+}$state in ${ }^{124} \mathrm{Te}$ can be interpreted as the lowest state of the first excited family of intrinsic levels in the critical point symmetry. However, it should be mentioned that a few of the measured $B(E 2)$ and $B(M 1)$ values have a large uncertainty and the experimental data of $B\left(M 1,1_{1}^{+} \rightarrow 0_{2}^{+}\right)$is still missing. More investigations on the experiment and the theory are needed to focus on these aspects.

\section{Acknowledgments}

We are grateful to Prof. CaiWan Shen, and Dr. XiaoBao Wang of Huzhou University, Prof. YuXin Liu of Peking University, and Prof. GuiLu Long of Tsinghua University for their

helpful discussions and good suggestions. This work is supported by the National Natural Science Foundation of China under grant numbers 11475062, 11747312 and 11147148.

[1] P. E. Garrett, J. L. Wood, and S. W. Yates, Phys. Scr., 93: 063001 (2018)

[2] L. C. He, Y. Zheng, L. H. Zhu et al, Chin. Phys. C, 41: 044003 (2017)

[3] S. F. Hicks, J. R. Vanhoy, P. G. Burkett, B. R. Champine, S. J. Etzkorn P. E. Garrett, S. W. Yates, and Minfang Yeh, Phys. Rev. C, 95: 034322 (2017)

[4] P. E. Garrett, J. Phys. G: Nucl. Part. Phys., 43: 084002 (2016)

[5] H. Sabria, Z. Jahangirib, M. A. Mohammadia, Nucl. Phys. A, 946: 11 (2016)

[6] M. Saxena, R. Kumar, A. Jhingan, S. Mandal, A. Stolarz, A. Banerjee, R. K. Bhowmik, S. Dutt, J. Kaur, V. Kumar, M. Modou Mbaye, V. R. Sharma, and H.-J. Wollersheim, Phys. Rev. C, 90: 024316 (2014)

[7] K. Heyde, Rev. Mod. Phys., 83: 1467 (2011)

[8] T. Kibédi, and R. H. Spear, At. Data Nucl. Data tables, 89: 77 (2005) 
[9] R. M. Clark, M. Cromaz, M. A. Deleplanque, M. Descovich, R. M. Diamond, P. Fallon, I. Y. Lee, A. O. Macchiavelli, H. Mahmud, E. Rodriguez-Vieitez, F. S. Stephens, and D. Ward, Phys. Rev. C, 69: 064322 (2004)

[10] D. G. Ghită, G. Căta-Danil, D. Bucurescu, I. Căta-Danil, M. Ivascu, C. Mihai, G. Suliman, L. Stroe, T. Sava and N. V. Zamfir, Int. J. Mod. Phys. E, 17: 1453 (2008)

[11] E. Guliyev, A. A. Kuliev, P. von Neumann-Cosel, and A. Richter, Phys. Lett. B, 532: 173 (2002)

[12] F. Iachello, Phys. Rev. Lett., 85: 3580 (2000)

[13] F. Iachello, Phys. Rev. Lett., 87: 052502 (2001)

[14] D. Bonatsos, D. Lenis, D. Petrellis, P. A. Terziev, and I. Yigitoglu, Phys. Lett. B, 621: 102 (2005)

[15] Y. Zhang, F. Pan, Y. X. Liu, Y. A. Luo, and J. P. Draayer, Phys. Rev. C, 96: 034323 (2017)

[16] F. Iachello, and A. Arima, The Interacting Boson Model (Cambridge, England:Cambridge University Press, 1987)

[17] M. A. Caprio, and F. Iachello, Ann. Phys., 318: 454 (2005)

[18] P. Cejnar, and J. Jolie, Prog. Part. Nucl. Phys., 62: 210 (2009)

[19] P. Cejnar, J. Jolie, and R. F. Casten, Rev. Mod. Phys., 82: 2155 (2010)

[20] T. Naz, G. H. Bhat, S. Jehangir, S. Ahmad, and J. A. Sheikh, Nucl. Phys. A, 979: 1 (2018)

[21] T. Otsuka, Hyperfine interactions, 75: 23 (1992)

[22] Y. Zhang, F. Pan, Y. X. Liu, Y. A. Luo, and J. P. Draayer, Phys. Rev. C, 90: 064318 (2014)

[23] N. Pietralla, P. von Brentano, and A. F. Lisetskiy, Prog. Part. Nucl. Phys., 60, 225 (2008)

[24] D. L. Zhang, S. Q. Yuan, and B. G. Ding, Chin. Phys. C, 39: 074102 (2015)

[25] Y. Zhang, Y. X. Liu, F. Pan, Y. Sun, and J. P. Draayer, Phys. Lett. B, 732: 55 (2014)

[26] A. Giannatiempo, A. Nannini, A. Perego, P. sona, M. J. G. Borge, K. Riisager, O. Tengblad, and ISOLDE Collaboration, Phys. Rev. C, 47: 521 (1993)

[27] D. L. Zhang, and C. F. Mu, Chin. Phys. C, 47: 034101 (2018)

[28] J. R. Vanhoy, J. A. Tanyi, K. A. Crandell, T. H. Churchill, S. F. Hicks, M. C. Burns, P. A. Roddy, N. V. Warr, T. B. Brown, and S. R. Lesher, Phys. Rev. C, 69: 064323 (2004)

[29] S. J. Robinson, W. D. Hamilton, and D. M. Snelling, J. Phys. G: Nucl. Part. Phys., 9: 961 (1983)

[30] J. Kern, P. E. Garrett, J. Jolie, and H. Lehmann, Nucl. Phys. A, 593: 21 (1995) 
[31] S. Pascu, N. V. Zamfir, Gh. Căta-Danil, and N. Mărginean, Phys. Rev. C, 81: 054321 (2010)

[32] J. Rikovska, N. J. Ston, P. M. Walker, and W. B. Walters, Nucl. Phys. A, 505: 145 (1989)

[33] N. Warr, S. Drissi, P. E. Garrett, J. Jolie, J. Kem, H. Lehmann, S. J. Mannanal, and J. P. Vorlet, Nucl. Phys. A, 636: 379 (1998)

[34] A. Subber, W. D. Hamilton, P. Park, and K. Kumar, J. Phys. G: Nucl. Part. Phys., 13: 161 (1987)

[35] H. Dejbakhsh, D. Latypov, G. Ajupova, and S. Shlomo, Phys. Rev. C, 46: 2326 (1992)

[36] D. L. Zhang, and B. G. Ding, Sci. China-Phys. Mech. Astron., 57: 447 (2014)

[37] D. L. Zhang, and C. F. Mu, Chin. Phys. Lett., 33: 102102 (2016)

[38] N. Pietralla, T. Mizusaki, P. von Brentano, R. V. Jolos, T. Otsuka, and V. Werner, Phys. Rev. C, 57: 150 (1998)

[39] T. Otsuka, and N. Yoshida, Program NPBOS, JAER-M Report, No.85 (1985)(unpublished)

[40] T. von Egidy, H.-F. Wirth, I. Tomandl, and J. Honzátko, Phys. Rev. C, 74: 034319 (2006)

[41] K. Nomura, T. Otsuka, N. Shimizu, and L. Guo, Phys. Rev. C, 83: 041302 (2011)

[42] K. Nomura, T. Otsuka, and P. V. Isacker, J. Phys. G: Nucl. Part. Phys., 43: 024008 (2016)

[43] D. L. Zhang, and C. F. Mu, Sci. China-Phys. Mech. Astron., 60: 042011 (2017)

[44] J. Rikovska, N. J. Stone, P. M. Walker, and W.B. Walters, Nucl. Phys. A, 505: 145(1989)

[45] C. Doll, H. Lehmann, H.G. Börner, and T. von Egidy, Nucl. Phys. A, 672: 3(2000)

[46] S. F. Hicks, J. R. Vanhoy, and S. W. Yates, Phys. Rev. C, 78: 054320 (2008)

[47] K.-H. Kim, A. Gelberg, T. Mizusaki, T. Otsuka, and P. von Brentano, Nucl. Phys. A, 604: $163(1996)$

[48] E. Elhami, J. N. Orce, S. Mukhopadhyay, S. N. Choudry, M. Scheck, M. T. Mcellistrem, and S. W. Yates, Phys. Rev. C, 75: 011301 (2007)

[49] D. L. Zhang, and C. F. Mu, Sci. China-Phys. Mech. Astron., 59: 682012 (2016)

[50] G. Mardirosian, and N. M. Steward, Z. Phys. A, 315: 213 (1984)

[51] R. F. Casten, and N.V. Zamfir, Phys. Rev. Lett., 85: 3584 (2000)

[52] R. F. Casten, D. D. Warner, Rev. Mod. Phys., 60: 389 (1988)

[53] F. Iachello, and N. V. Zamfir, Phys. Rev. Lett., 92: 212501 (2004)

[54] Y. Zhang, Z. F. Hou, and Y. X. Liu, Phys. Rev. C, 76: 011305(R) (2007)

[55] D. Bonatsos, E. A. McCutchan, R. F. Casten, and R. J. Casperson, Phys. Rev. Lett., 100: $142501(2008)$ 
[56] J. Stachel, P. Van Isacker, and K. Heyde, Phys. Rev. C, 25: 650 (1982)

[57] P. Van Backer, K. Heyde, J. Jolie, and A. Sevrin, Ann. Phys., 171: 253 (1986)

[58] A. Sevrin, K. Heyde, and J. Jolie, Phys. Rev. C, 36: 2621 (1987)

[59] R. Georgii, T. von Egidy, J. Klora, H. Lindner, U. Mayerhofer, J. Ott, W. Schauer, P. von Neumann-Cosel, A. Richter, C. Schlegel, R. Schulz, V. A. Khitrov, A. M. Sukhovoj, A. V. Vojnov, J. Berzins, V. Bondarenko, P. Prokofjevs, L. J. Simonova, M. Grinberg, and Ch. Stoyanov, Nucl. Phys. A, 592: 307 (1995)

[60] S. F. Hicks, J. C. Boehringer, N. Boukharouba, C. Fransen, S. R. Lesher, J. M. Mueller, J. R. Vanhoy, and S. W. Yates, Phys. Rev. C, 86: 054308 (2012)

[61] D. L. Zhang, and C. F. Mu, Sci. China-Phys. Mech. Astron, 61: 012012 (2018)

[62] K. Nomura, R. Rodríguez-Guzmán, and L. M. Robledo, Phys. Rev. C, 95: 064310 (2017)

[63] A. Leviatan, and D. Shapira, Phys. Rev. C, 93: 051302 (2016)

[64] J. L. Wood, E. E. Zganjar, C. E. Coster, and K. Heyde, Nucl. Phys. A, 651: 323 (1999)

[65] A. Giannatiempo, A. Nannini, A. Perego, and P. Sona, Phys. Rev. C, 44: 1844 (1991)

[66] O. Möller, N. Warr, J. Jolie, A. Dewald, A. Fitzler, A. Linnemann, K. O. Zell, P. E. Garrett, and S. W. Yates, Phys. Rev. C, 71: 064324 (2005)

[67] C. Qi, Phys. Rev. C, 94: 034310 (2016)

[68] M. Doncel, T. Bäck, C. Qi, D. M. Cullen, D. Hodge, B. Cederwall, M. J. Taylor, M. Procter, M. Giles, K. Auranen, T. Grahn, P. T. Greenlees, U. Jakobsson, R. Julin, S. Juutinen, A. Herzán̆, J. Konki, J. Pakarinen, J. Partanen, P. Peura, P. Rahkila, P. Ruotsalainen, M. Sandzelius, J. Sarén, C. Scholey, J. Sorri, S. Stolze, and J. Uusitalo, Phys. Rev. C, 96: 051304 (2017) 